\title{
BEAM INSTABILITIES MEASUREMENT AND CURES AT HLS*
}

\author{
Y.L. Yang", J.H. Wang, Z.P. Liu, W.M. Li, B.G. Sun, Kai Zheng, Z.R. Zhou, Y.B. Chen, L.J. Huang \\ NSRL, University of Science and Technology of China, Hefei, Anhui 230029, P.R.China
}

\section{Abstract}

In Hefei Light Source (HLS), coupled-bunch instabilities are major limiting factors in achieving higher beam intensity while maintaining good beam quality. To Measure and suppress beam instabilities, turn-by-turn (TBT) measurement and bunch-by-bunch $(\mathrm{BxB})$ measurement \& feedback system are under commission [1][2]. The design of the two systems and primary experiment results is presented. Measurement and detail analysing results in injection status will also be shown.

\section{INTRODUCTION}

HLS is a synchrotron light source injecting in the energy of $200 \mathrm{MeV}$ and operating in $800 \mathrm{MeV}$, which operates with 45 bunches in $204.016 \mathrm{MHz}$ RF. The circumference of electron storage ring is 66 meters. A multi-cycle multi-turn injection system is used for current accumulation. The maximum electron current is presently limited to about $250 \mathrm{~mA}$ due to multi-bunch instabilities. Those are observed especially at injection and energy ramp by turn-by-turn and bunch-by-bunch measurement system. The implementation of a transversal bunch-bybunch feedback system is necessary.

\section{SYSTEM DESCRIPTION}

\section{TBT Measurement System}

HLS Turn-by-turn system consists of front end pick-up electrodes mounted in a skew $45^{\circ}$, Log-ratio electronics, timing system, and data acquisition system. The log-ratio processor works at $408 \mathrm{MHz}$ which is $2 *$ RF of HLS[3].

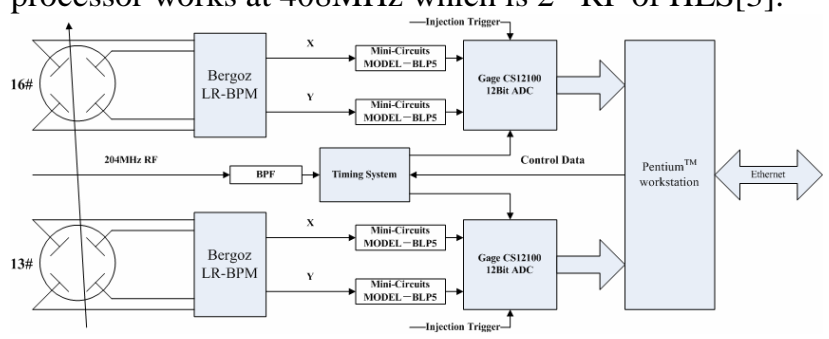

Figure 1: Overview of HLS Turn-by-Turn Measurement System

The data acquisition system includes two $\mathrm{GaGe}^{\mathrm{TM}}$ 12Bit ADCs in the ADVANTECTM Industrial Computer 610. The Timing System divides down the RF signal. And two in-phase TTL signals (have same frequency of $4.533 \mathrm{MHz}$ ) provides to each of the ADC as the external clock. Further more, the time delay between two TTL signals can be finely adjusted by computer over network and the delay range is from $0.5 \mathrm{~ns}$ to $220 \mathrm{~ns}$.

\footnotetext{
* Supported by National Natural Science Project (10175063) and National Natural Science Key Project (10535040)

\#ylyang@mail.ustc.edu.cn
}

06 Instrumentation, Controls, Feedback \& Operational Aspects

\section{BxB Measurement and Feedback System}

The transverse bunch-by-bunch feedback system of HLS is based on designs for PLS[4]. An optical-fiber two-tap FIR filter[5] and a new feedback cavity have been developed for this system. This system is integrated with longitudinal oscillation measurement system, fast vector control, fiber notch filter and bunch current detection system.

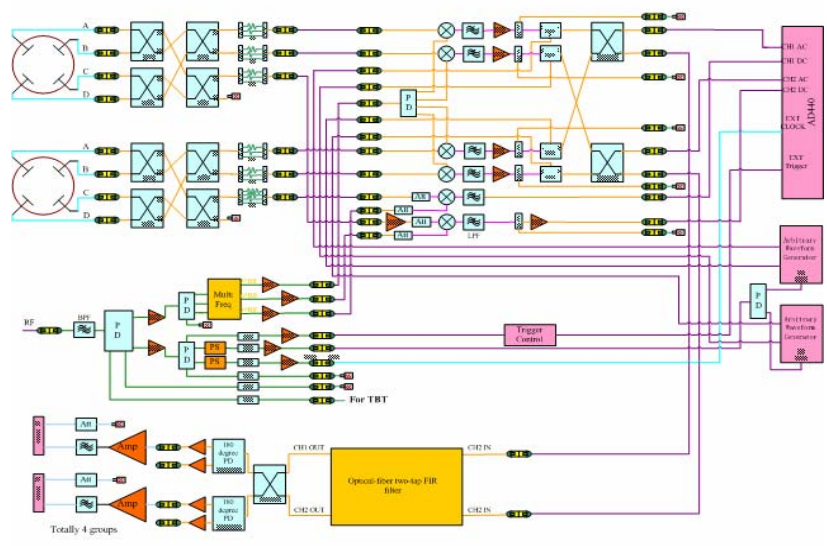

Figure 2: Overview of HLS BxB feedback and measurement system.

The $\mathrm{BxB}$ measurement system is dedicated to observe the beam instability and works as a part of the anolog transverse $\mathrm{BxB}$ feedback system in HLS. The system works at $612 \mathrm{MHz}\left(3 * \mathrm{f}_{\mathrm{RF}}\right)$, the longitudinal one works at $1224 \mathrm{MHz}\left(6 * f_{\mathrm{RF}}\right)$, both of them with $100 \mathrm{MHz}$ bandwidth. A high speed 12-bit digitizer, up to 400 MSPS (Million Samples Per Second) and simultaneous in two channels, is installed in a PXI (PCI eXtensions for Instrumentation) desktop. An in-phase FRF signal is used as the external clock of the ADC (Anolog-to-Digital Converter), and an in-phase Revolution Frequency signal $(4.533 \mathrm{MHz})$ is connected with the External Trigger of the ADC[6][7]

\section{BXB FEEDBACK AND MEASUREMENT SYSTEM TIME-SETTING TUNE}

With the using of new optical-fiber two-tap FIR filter and new feedback cavity, setting of the timing of feedback systems need readjustment. For better timing and bunch identification the number of bunches in the storage ring was reduced to single bunch in one train. Because of multi-cycle multi-turn injection system, a resonant knockout generator was used, which allowed the deleting of discrete bundles in the train after the injection is completed. Figure 3 show the single bunch signals recorded by an oscilloscope: $\mathrm{C} 1$ is bunch position signal, and $\mathrm{C} 2$ is the amplified feedback signal and the signal induced in the feedback cavity strip-line by the beam.

T05 Beam Feedback Systems 


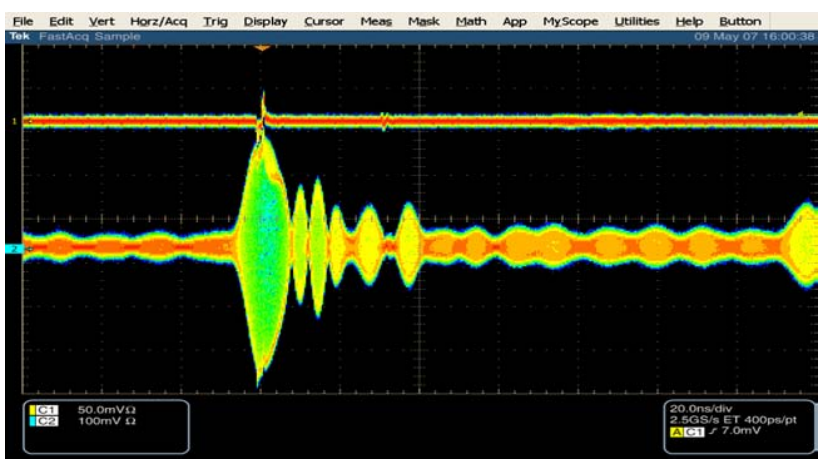

Figure 3: Beam Position signal and feedback signal from kicker cavity of Timing adjustment

\section{MEV INJECTION INSTABILITIES MEASUREMENT}

One aim of building this feedback system is to improve the maximum accumulation electron current at injection status. So, recording the bunch-by-bunch beam position data is very important when electron current reach the limit and not accumulate anymore. With turn-by-turn and bunch-by-bunch measurement system, we can observe injection instabilities at real time and can know whether multi-bunch instabilities are effectively cured.

The bunch-by-bunch transverse position tracing shows in Figure 4, from which injection damping can be clearly recorded. Injection signal is provided to measurement system as trigger.

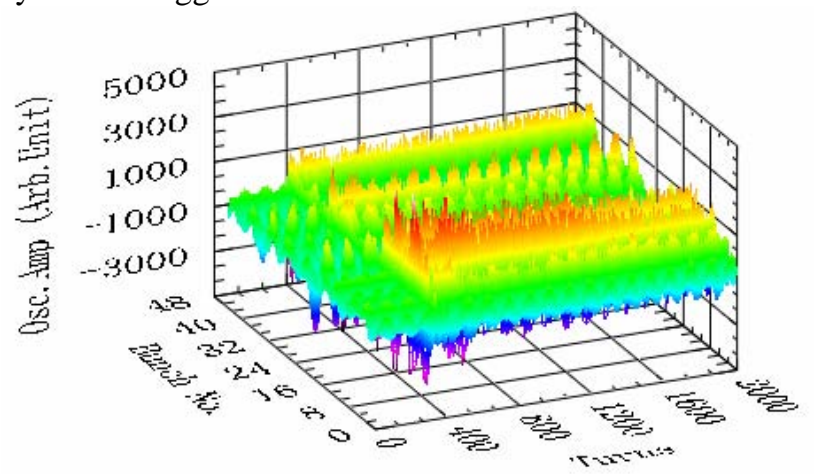

Figure 4: Bunch transverse position tracing at injection

From data of figure 4, we can get the frequency map, phase information, bunch motion in mode space of each bunch.

While injecting, longitudinal and transverse oscillation are much intense. Detail analysing result of bunch-bybunch transverse position tracing is showed from figure 5 to figure 8 .

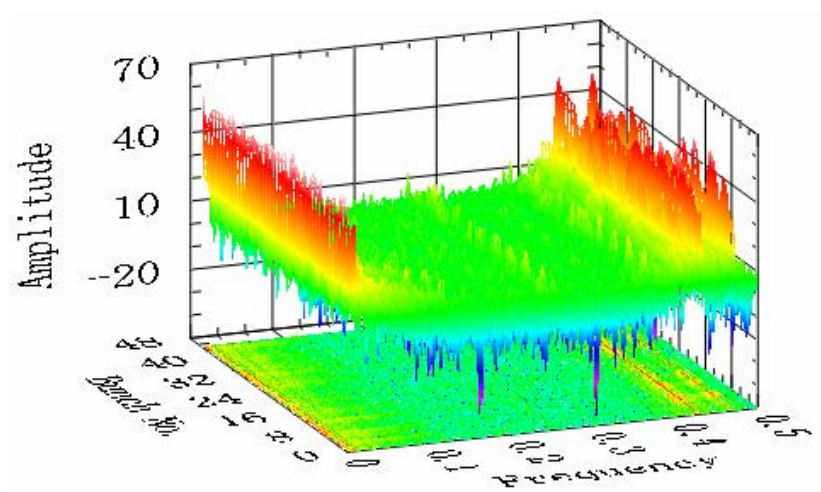

Figure 5: Bunch Frequency tracing

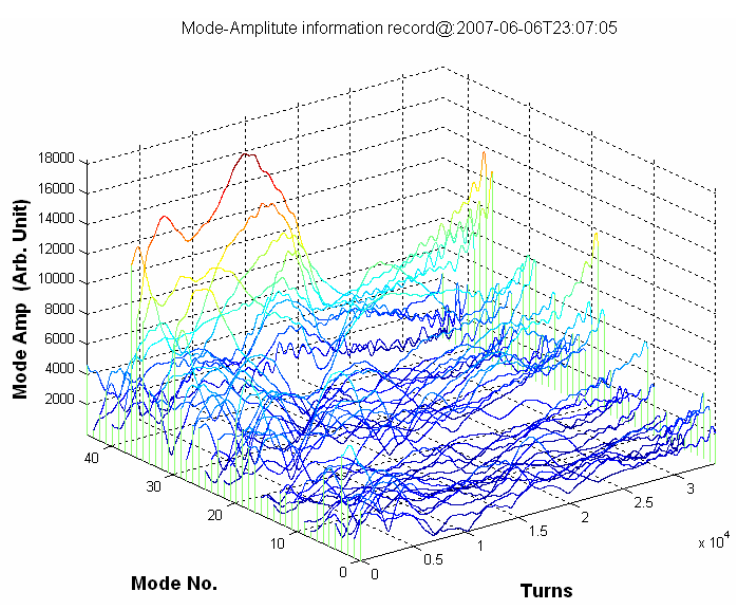

Figure 6: Mode tracing in time domain of $200 \mathrm{MeV}$ injection status

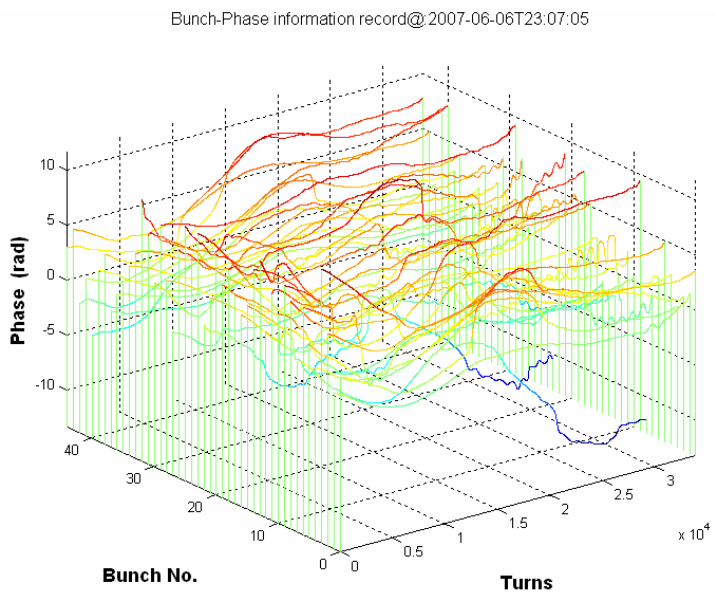

Figure 7: Phase tracing in time domain of $200 \mathrm{MeV}$ injection status 


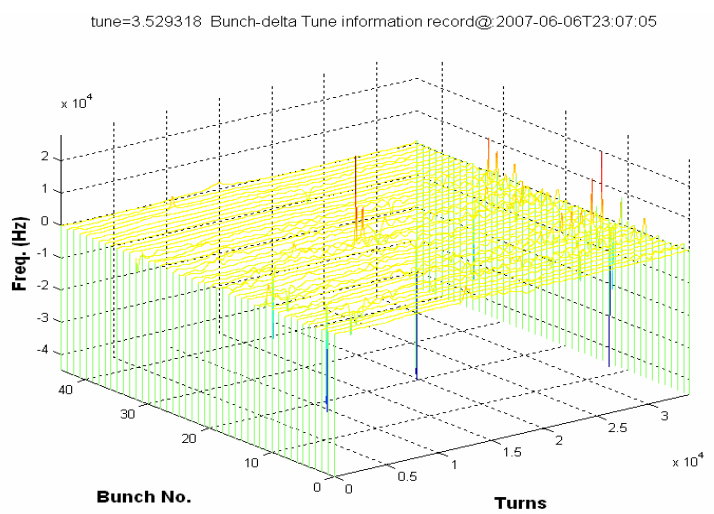

Figure 8: Oscillation frequency

\section{MEV FEEDBACK READJUSTMENT}

To adjust the feedback phase, two BPM are selected to just the feedback signal. DBM (double balance mixer) can supply not only a positive adjustable attenuate factor, but also a negative adjustable attenuate factor. It is a great upgrade of the flexible of this feedback system. Compare with the 90 degree adjust range, this method can support 360 degree feedback signal vector calculating

\section{Feedback Phase Readjustment}

Figure 9 and 10 show the Synchrotron beam profile and corresponding bunch-by-bunch frequency spectrum. With in-phase signal, the feedback system is used to cure the beam instabilities, and anti-phase is to excite.

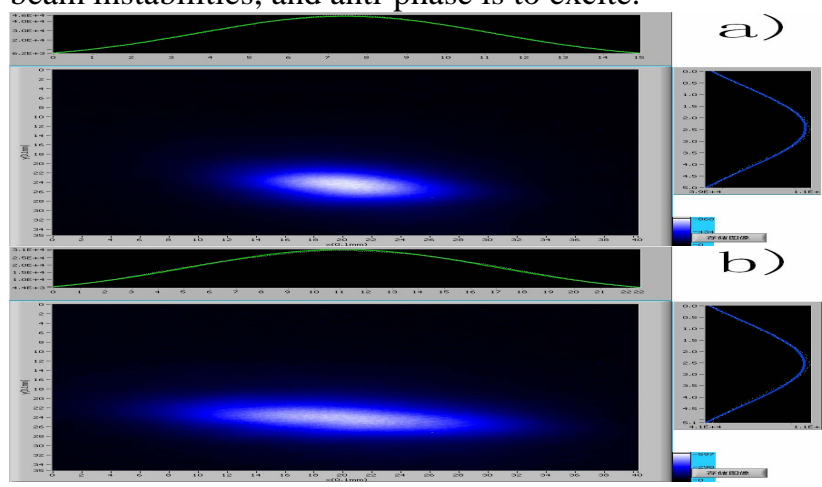

Figure 9: Synchrotron beam profile, part a) feedback off status, part b) feedback on with anti-phase

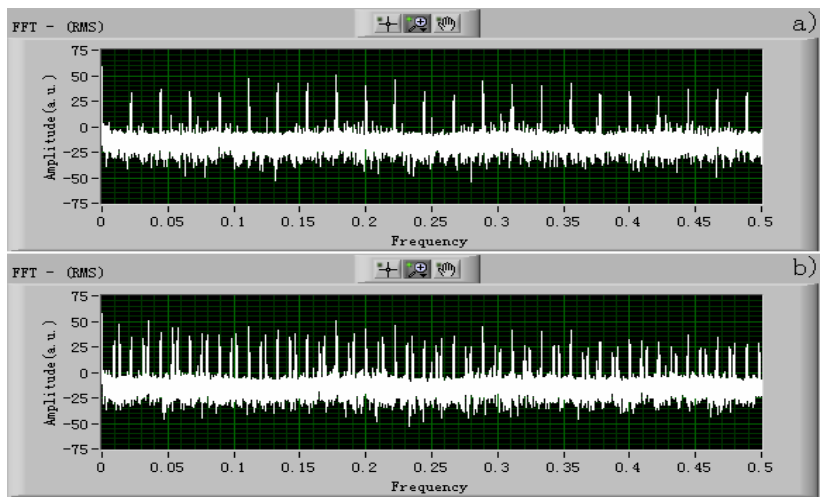

Figure 10: frequency spectrum, part a) feedback off status, part b) feedback on with anti-phase

06 Instrumentation, Controls, Feedback \& Operational Aspects

\section{EXPERIMENT CONCLUSION}

To now, with the optical-fiber two-tap FIR filter and a new feedback cavity, we only have readjusted time-setting and feedback phase at $800 \mathrm{MeV}$ operation status, have measured the bunch-by-bunch tracing data at $200 \mathrm{MeV}$ injection. The next we need to do is readjust the feedback phase at $200 \mathrm{Mev}$ injection status, and cure the coupled bunch instabilities at injection and increase the current to rather high values

\section{ACKNOWLEDGMENTS}

The authors would like to present their thanks to Hongliang $\mathrm{Xu}$, Lin Wang, Fengguan Yao and Shancai Zhang of HLS for direct contributions of technical expertise. We also thank Li Ma, Jianshe Cao and Junhui Yue of BEPCII for their numerous thoughtful discussions.

\section{REFERENCES}

[1] Kai Zheng, J. H. Wang, J. H. Liu, "Bunch Tracing by Bunch by Bunch Measurement System in HLS", PAC'05, Knoxville, Tennessee, 2005.

[2] WANG Jun-hua, LIU Jian-hong and ZHENG Kai, "Design of bunch-by-bunch measuring and transverse feedback system at HLS", High Power and Particle Beams, 2006(02).

[3] Yang Yong-liang, WANG Jun-hua, LIU Zu-ping, "Phase-space Measurement of Hefei Light Source," High Power Laser and Particle Beams, 2006, 18(8)

[4] J. Y. Huang, M. K. Park, M. Kwon and M. Yoon, "TRANSVERSE BEAM FEEDBACK SYSTEM IN THE PLS', APAC'98, P2350-2352

[5] Yan Yin, Xiuge Che, "Optical-Fiber Two-Tap FIR Filter for Storage Ring Transverse Feedback System", Proceedings of PAC07

[6] Jianhong Liu, "The development of Bunch-by-Bunch measurement system and the research of instability in HLS," thesis for Doctor Degree of University of Science and Technology of China

[7] Kai Zheng, J. H. Wang, Z. P. Liu, "BUNCH-BYBUNCH MEASUREMENT AND FEEDBACK SYSTEM OF HLS," Proceedings of PAC07

[8] Mario Serio, "Multi bunch Instabilities and Cures", PAC99, New York, 1999. 